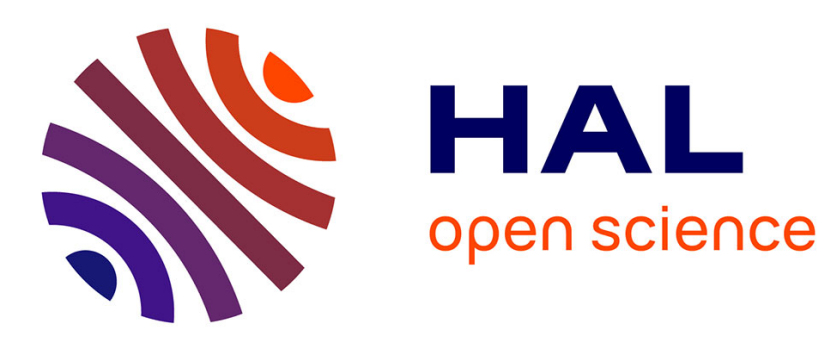

\title{
Mode de glissement des dislocations (010)[001] dans les feldspaths alcalins. methr et modélisation.
}

\author{
Yunlin Jacques Zheng, M. Gandais, M. Heggie
}

\section{To cite this version:}

Yunlin Jacques Zheng, M. Gandais, M. Heggie. Mode de glissement des dislocations (010)[001] dans les feldspaths alcalins. methr et modélisation.. Revue de Physique Appliquée, 1988, 23 (4), pp.669-669. 10.1051/rphysap:01988002304066900 . jpa-00245818

\section{HAL Id: jpa-00245818 https://hal.science/jpa-00245818}

Submitted on 1 Jan 1988

HAL is a multi-disciplinary open access archive for the deposit and dissemination of scientific research documents, whether they are published or not. The documents may come from teaching and research institutions in France or abroad, or from public or private research centers.
L'archive ouverte pluridisciplinaire HAL, est destinée au dépôt et à la diffusion de documents scientifiques de niveau recherche, publiés ou non, émanant des établissements d'enseignement et de recherche français ou étrangers, des laboratoires publics ou privés. 


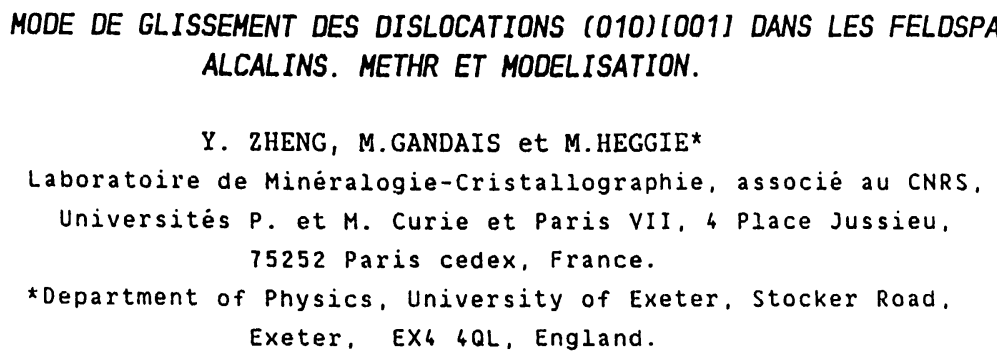

Les feldspaths sont des aluminosilicates de formule chimique $\mathrm{MT}_{4}{ }_{8}$ où $M$ représente un cation $\mathrm{K}$, $\mathrm{Na}$, ou $\mathrm{Ca}$ et $\mathrm{T}$ représente un atome $\mathrm{Al}$ ou $\mathrm{Si}$ avec $\mathrm{Al} / \mathrm{Si}=1 / 3$ dans les feldspaths alcalins et $1 / 1$ dans les feldspaths purement calciques. Ce sont les minéraux les plus abondants de la croûte terrestre. D'un point de vue structural, ce sont des tectosilicates (commme le quartz), c'est-àdire qu'ils sont formés d'une charpente tridimensionnelle de tétradèdres $\mathrm{TO}_{4}$ reliés entre eux par leurs quatre sommets. La charpente feldspathique $a$ une configuration particulière: elle possède des doubles chaînes de tétraèdres en forme de "vilebrequin" dans la direction $\vec{a}$ de la maille, les doubles chaines étant reliées entre elles par des liaisons dans les directions $\vec{b}$ et $\vec{c}$. Les cations sont logés dans de larges cavités entre les doubles chaines. Dans une telle structure, tout glissement de dislocation implique la rupture de liaisons fortes $T-0-T$ et, pour une large part, les modèles élaborés pour les semiconducteurs à liaisons tétraèdriques peuvent servir de référence.

Les échantillons examinés en METHR sont des monocristaux de sanidine et d'othose déformés expérimentalement. Ce sont des feldspaths alcalins à forte proportion de potassium. Ils sont de symétrie monoclinique $(\mathrm{C} 2 / \mathrm{m})$. Les expériences de déformation ont été réalisées sous pression de confinement $\mathrm{P}=1500 \mathrm{MPa}$ à la température $\mathrm{T}=700^{\circ} \mathrm{C}$ $\left(T_{f} \sim 1100^{\circ} \mathrm{C}\right)$ [1]. Les dislocations $(010)[001]$ sont actives à partir de la température de transition fragile-ductile $\left(T / T_{f} \sim 0,7\right)$ et au-dessus. Elles sont importantes dans nos échantillons déformés en laboratoire aussi bien que dans d'autres types de feldspaths déformés dans la nature. $L^{\prime}$ étude par microscopie électronique en transmission à haute résolution (METHR) combinée à une analyse structurale a permis d'affiner nos concepts sur le glissement des dislocations dans ces minéraux.

En METHR, les images de segments mixtes [100] et [101] indiquent que les dislocations (010)[001] sont dissociées dans leur plan de glissement en deux partielles séparées par un défaut plan (010)[001]/2. La séparation entre les partielles est de l'ordre de 15 mailles ou $12 \mathrm{~nm}$, ce qui donne pour l'énergie du défaut plan une valeur $\gamma \sim 0,06 \mathrm{~J} / \mathrm{m}^{2}$ [2][3].

Dans la maille des feldspaths il y a deux positions possibles pour un plan de glissement (010), de coordonnées respectives $y=0$ et $y=1 / 4$. Le premier plan passe entre les doubles chaines et coupe un minimum de liaisons T-O-T ( 2 par maille). Pour cette raison on pensait initialement que c'était la position du plan de glissement. Le second plan passe au milieu des doubles chaines et coupe deux fois plus de liaisons ( 4 par maille). L'analyse structurale montre qu'un défaut plan (010)[001]/2 sans liaisons pendantes -qui coûtent cher en énergie dans ces matériaux isolants- ne se conçoit que dans la seconde position $y=1 / 4$, c'està-dire dans la double chaine. Cette position nécessite de couper un plus grand nombre de liaisons mais elle préserve les sites cationiques et de plus, permet des reconstructions des liaisons $T-0-T$ au coeur des dislocations.

L'énergie du défaut plan a été estimée en utilisant pour le potentiel cristallin un modèle de Keating qui tient compte des interactions entre premiers voisins pour les atomes $T$ et 0 de la charpente aluminosilicatée. On trouve $r \sim 0,38 \mathrm{~J} / \mathrm{m}^{2}$. Cette valeur est supérieure à la valeur expérimentale, ce qui s'explique par le fait qu'avec l'approximation utilisée, on comptabilise dans la déformation toute différence entre les angles $0-T-0$ et ceux du tétraèdre régulier ains $i$ que tout écart des angles $T-0-T$ à la valeur moyenne, y compris ceux qui existent dans la structure du cristal parfait à cause des cations. Compte. tenu de cette approximation, l'écart entre les valeurs mesurées et calculées (moins d'un ordre de grandeur) est très satisfaisant [4].

\section{Références :}

[1] WILLAIME C., CHRISTIE J. M. and KOVACS M.-P. (1979). Bull. Mineral., 102, 168-177.

[1] ZHENG Y. et GANDAIS M., (1987). Bull. Mineral., 110, 15-24.

[2] ZHENG Y. and GANDAIS M., (1987). Phil. Mag., sous presse.

[3] HEGGIE M. and ZHENG Y., (1987). Phil. Mag. sous presse. 